\title{
Examining the stress-burnout relationship: The mediating role of negative thoughts
}

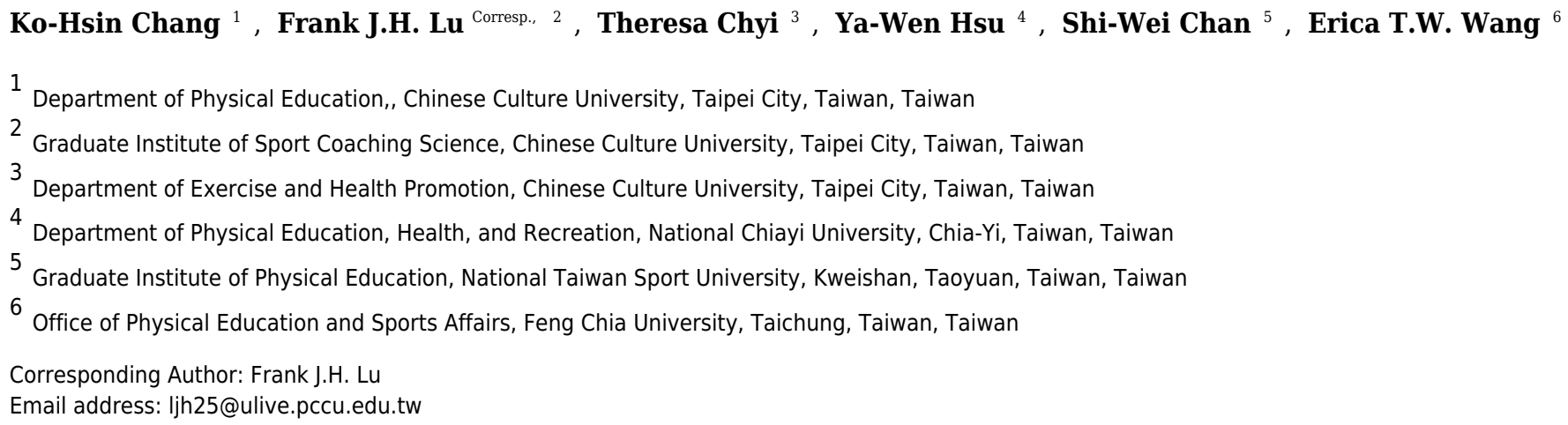

Background: Using Smith's (1986) cognitive-affective model of athletic burnout as a guiding framework, the purpose of this study was to examine the relationships among athletes' stress in life, negative thoughts, and the mediating role of negative thoughts on the stress-burnout relationship. Methods: $A$ total of 300 college student-athletes (males = 174 ; females $=126, M_{\text {age }}=20.43$ y, $S D=1.68$ ) completed the College Student Athlete's Life Stress Scale (CSALSS; Lu et al., 2012), Automatic Thoughts Questionnaire (ATQ; Hollon \& Kendall, 1980), and Athlete Burnout Questionnaire (ABQ; Raedeke \& Smith, 2001). Results: Correlational analyses found that two types of life stress and four types of negative thoughts correlated with burnout. Additionally, hierarchical regression analyses found that four types of negative thoughts partially mediated the stress-burnout relationship. Discussion: We concluded that an athlete's negative thoughts play a pivotal role in predicting athletes' stress-burnout relationship. Future study may examine how irrational cognition influences athletes' motivation and psychological well-being. 
Examining the Stress-Burnout Relationship: \& Erica T.W. Wang ${ }^{4}$

${ }^{4}$ Feng Chia University

12 Correspondence concerning this article should be addressed to Frank J.H. Lu

13 Institute: Department of Physical Education, Chinese Culture University

14 Phone: 1-886-933-029265

15 Fax: 1-886-3-3280596

16 E-mail: ljh25@ulive.pccu.edu.tw

\section{Ko-Hsin Chang ${ }^{1}$}

21 Department of Physical Education, Chinese Culture University

22 Tel: 1-886-2-28610511 ext .43226 (office) 
23 E-mail: kathy0620@gmail.com

25 Frank J. H. Lu*1

26 Department of Physical Education, Chinese Culture University

27 Tel: 1-886-2-28610511 ext .43226 (office)

28 E-mail: 1jh25@ulive.pccu.edu.tw

29 Theresa Chyi ${ }^{1}$

30 Department of Exercise and Health Promotion, Chinese Culture University

31 Tel: 1-886-2-28610511 ext .43831 (office)

32 E-mail: tcc@ulive.pccu.edu.tw

33

\section{Ya-Wen Hsu ${ }^{2}$}

35 Department of Physical Education, Health, and Recreation, National Chia-Yi University

36 Tel: TEL: +886-5-2717000 FAX: +886-5-2717095

37 E-mail: tgieva@gmail.com

39 Shi-Wei Chan ${ }^{3}$

40 Graduate Institute of Physical Education, National Taiwan Sport University

41 Tel: 1-886-3-3283201 ext. 8532

42 E-mail: jswm4zp@gmail.com

43

44 Erica T.W. Wang ${ }^{4}$

45 Office of Physical Education and Sports Affairs, Feng-Chia University

46 Tel: 1-886-4-24517250 ext. 5985 598

47 E-mail: tingwen830@hotmail.com 


\section{Abstract}

53 Background: Using Smith's (1986) cognitive-affective model of athletic burnout as a guiding

54 framework, the purpose of this study was to examine the relationships among athletes' stress in

55 life, negative thoughts, and the mediating role of negative thoughts on the stress-burnout

56 relationship. Methods: A total of 300 college student-athletes (males $=174$; females $=126, M_{\text {age }}$

$57=20.43$ y, $S D=1.68)$ completed the College Student Athlete's Life Stress Scale (CSALSS; Lu et al., 2012), Automatic Thoughts Questionnaire (ATQ; Hollon \& Kendall, 1980), and Athlete Burnout Questionnaire (ABQ; Raedeke \& Smith, 2001). Results: Correlational analyses found that two types of life stress and four types of negative thoughts correlated with burnout. Additionally, hierarchical regression analyses found that four types of negative thoughts partially mediated the stress-burnout relationship. Discussion: We concluded that an athlete's negative thoughts play a pivotal role in predicting athletes' stress-burnout relationship. Future study may examine how irrational cognition influences athletes' motivation and psychological well-being. 


\section{Introduction}

67 Being successful in sports brings fame and monetary rewards to athletes, but the costs of

68 athletic success are high. To achieve this, athletes not only have to engage in sports at a very

69 young age but also need to participate in intensive training every day and compete year-round

70 (Gould \& Whitley, 2009). Research has suggested that intensive involvement in sports is not

71 beneficial. One of the potential negative outcomes is burnout. Burnout is a complex psycho-

72 physiological symptom characterized by physical and emotional exhaustion, de-evaluation in the

73 sport, and reduced sense of accomplishment (Raedeke \& Smith, 2004). Although the prevalence

74 of burnout has been minimal in both female athletes $(1 \%-9 \%)$ and male athletes $(2 \%-6 \%)$

75 (Gustafsson, Kentta, Hassmen \& Lindquist, 2007), studies have indicated that the burnout

76 experience is detrimental to an athlete's psychological well-being (Franciscoa et al., 2016; Gould

77 et al., 1997), resulting in depression (Francisco et al., 2016), increasing interpersonal difficulties

78 (Smith, 1986), and triggering drop-out (Gould \& Whitley, 2009). 
79 To explain the process of athlete burnout, Smith (1986) proposed a "cognitive-affective

80 model of athletic burnout," which contends that burnout is a reaction to chronic stress and it

81 comprises situational, cognitive, physiological, and behavioral components that progress in four

82 predicted stages. The first stage is triggered by the athletes' perceived situational demands (e.g.,

83 high conflicting demands, overload training, parental expectations, or coaches' pressures). The

84 second stage involves cognitive appraisal to interpret these demands. In particular, athletes at this

85 stage will appraise the balance between challenges and resources with potential consequences.

86 When athletes perceive that demands surpass personal resources and consequences will be severe,

87 the process will move to the third stage, which involves physiological and psychological

88 responses, such as anxiety, tension, insomnia, and illness. Finally, physiological and

89 psychological responses lead to burnout (i.e., rigid and inappropriate behavior, decreased

90 performance, and withdrawal from the activity).

91 Based on the cognitive-affective model of athletic burnout, many studies attempt to

92 examine the association between an athlete's stress and burnout; and the mechanism underlying

93 the stress-burnout relationship. For example, Cresswell and Eklund (2006) interviewed 15 New 
94 Zealand professional rugby players and they found that these athletes had many negative

95 experiences, such as injury, competitions, performance demands, anti-rest culture, media

96 pressure, and training loadings associated with burnout. In addition, Gustafsson, Hassmen, Kenta,

97 and Johansson (2008) interviewed 11 Swedish athletes and found that multiple demands, such as

98 "too much sport," "lack of recovery," and "high expectations," were considered primary causes

99 of burnout. Recently, Francisco and colleagues (2016) investigated 453 Spanish athletes and

100 found that perceived stress accounted for $43 \%$ of the variance for burnout. Furthermore,

101 perceived stress and burnout jointly accounted for $50 \%$ of the variance in depression.

102 In addition, researchers have also examined the mechanisms underlying the stress-burnout

103 relationship. For example, Gustafsson and Skoog (2012) measured 217 participants for their

104 level of optimism, perceived stress, and burnout. They found perfectionism, perceived stress, and

105 burnout all correlated, and perceived stress fully mediated the optimism-burnout relationship.

106 Furthermore, when considering that hope is a positive buffer for stress-related burnout,

107 Gustafsson, Skoog, Podlog, Lundqvist, and Wagsson (2013) investigated 238 Swedish youth

108 soccer players and examined the relationships among hope, perceived stress, and burnout. They 
109 found that hope negatively correlated with perceived stress and all three dimensions of burnout.

110 Additionally, the relationship between hope and burnout was fully mediated by stress and

111 positive effects. Recently, Lu et al., (2016) adopted an interactive perspective to examine the

112 conjunctive effects of athletes' resilience and coaches' social support in moderating the stress-

113 burnout relationship. Results indicated that athletes' resilience correlated with the level of the

114 coaches' social support, and this effect moderated athletes' stress-burnout relationship.

115 Specifically, athletes' resilience interacted with coaches' informational/tangible social support in

116 moderating athletes' stress-burnout relationship in both high and low life stress situations.

117 Despite these efforts, it is unknown what role negative thoughts play in the stress-burnout

118 relationship. The study of negative thoughts can be traced back to Beck's (1976) works on

119 depressed patients. In his works, he proposed there are three key elements: negative views about

120 the world, negative views about the future, and view of the self, formed a negative cognition

121 triad model. Beck (Beck, 1976) contended that some individuals encounter major life events and

122 become depressed are because of cognitive distortion and irrational thoughts. In non-sports

123 settings, it was found that negative thoughts are not only linked to depression (Clark \& Goosen, 
124 2009), but they are also associated with pain/psychological distress (Gil et al., 1990), fatigue

125 (Arpin-Cribbie \& Cribbie, 2007), and a dysphoric mood (Stiles \& Gotestam, 1989).

126 Negative thoughts play a pivotal role in the cognitive-affective model of athletic burnout.

127 Specifically, Smith contends that when encountering environmental stressors, athletes engage in

128 a cognitive appraisal to evaluate whether these stressors are threatening or whether they can

129 handle them (Smith, 1986). Thus, the ways in which athletes appraise the stressors, both from

130 positive and negative perspectives, are worthy of investigation.

131 In sports, there are several types of negative thoughts. The first example is negative self-talk.

132 Self-talk is a cognitive strategy that athletes use to direct their attention or psych themselves up

133 (Hatzigeorgiadis et al, 2011). Literature has indicated that self-talk can be either negative or

134 positive, and it can influence athletes' affect, cognition, and performance (Chang et al. , 2014;

135 Zourbanos et al., 2010).

136 The second example is the negative expectation. Negative expectation refers to one's

137 expectation that something positive will not happen in the future. In sports, the negative

138 expectation is closely related to cognitive anxiety. For example, Martens and colleagues 
139 (Martens et al., 1990) conducted a series of studies to develop a state anxiety measure termed the

140 "Competitive State Anxiety Inventory-2" (CSAI-2). The CSAI-2 consists of three factors:

141 somatic anxiety, cognitive anxiety, and confidence. Cognitive anxiety consists of negative

142 expectations (e.g., I am concerned I may not do as well in this competition as I could). Empirical

143 studies found that negative expectations associated with anxiety and lowered overall

144 performance (Suhr \& Gunstad, 2004). Some other research also found negative expectations

145 were associated with poor sports injury recovery (Arvinen-Barrow et al., 2016).

146 The third example is self-defeating attribution. Self-defeating refers to attribute success as

147 external, unstable, or uncontrollable variables (e.g., others' help, luck, weather), while attribute

148 failure refers to internal, stable, and controllable reasons (e.g., ability, task, referee.) Athletes use

149 different explanations for their achievements and social events (Allen, 2012), such as explaining

150 failures and successes (e.g., Geringon \& Delloye, 2003), interpersonal interactions (e.g.,

151 Dimmock, Grove, \& Eklund, 2005), and injuries (e.g., Brewer, 1999). Though self-defeating

152 behavior is not often seen in males, it is frequently found in female athletes (e.g., Green \& 
153 Holeman, 2004). Research indicated that athletes' causal attributions influence their motivation,

154 affect, and performance (Allen, 2012).

155 Using the aforementioned theoretical and empirical background, the purpose of this study

156 was to examine the relationships among athletes' life stressors (both in sports and in general life),

157 negative thoughts (i.e., personal maladjustment, the desire for change, negative sense of self,

158 giving up, learned helplessness, and negative expectations), and burnout. We also examined the

159 mediating role of negative thoughts on the life stress-burnout relationship. Because past research

160 has indicated that negative life events precede negative thoughts (e.g., Lakey \& Tanner, 2013),

161 we hypothesized that athletes' life stress would positively correlate with negative thoughts.

162 Furthermore, because research has found that negative thoughts are associated with maladaptive

163 outcomes (e.g., Arpin-Cribbie \& Cribbie, 2007; Clark \& Goosen, 2009; Gil et al., 1990; Stiles \&

164 Gotestam, 1989), we hypothesized that negative thoughts would positively correlate with athlete

165 burnout. Moreover, because negative thoughts are likely related to athletes' life stress and

166 burnout, it was hypothesized that negative thoughts would play a mediating role in an athlete's

167 life stress-burnout relationship. The rationale was based on Baron and Kenny (1986, p. 1176), 
168 who suggested that a mediator is a third variable that explains how external physical events have

169 internal psychological significance.

171 Participants

172 Through a convenience sampling, 300 student-athletes $\left(\right.$ males $=174$; females $=126, M_{\text {age }}=$

$17320.43 \mathrm{y}, S D=1.68)$ from five colleges voluntarily participated in this study. During data

174 collection, all participants were engaged in their regular training seasons. All participants had

175 been participating in either team sports (basketball, softball, volleyball) or individual sports

176 (gymnastics, air pistol, tennis, track and field, table tennis, tug of war, archery, or golf) for an

177 average of $9.83 \mathrm{y}(\mathrm{SD}=3.42 \mathrm{y})$ of training and competition experiences. 


\section{Measures}

179 General Characteristics: We used a demographic questionnaire to collect information about

180 participants' age, gender, types of sports, training hours per day, training frequency per week,

181 and years of athletic experience.

182 Life Stressors: To measure participants' life stressors, we used the 24-item College Student-

183 Athletes Life Stress Scale (CSALSS; Lu et al, 2012) to evaluate situations that athletes encounter

184 in their daily life and in sports, which represent major stressors. These stressors all had two

185 major components: sport-specific stress (i.e., coach relationships, performance demand, sports

186 injuries, and training adaptation) and general life stress (i.e., family relationships, interpersonal

187 relationships, romantic relationships, academic requirements). For instance, on various questions,

188 such as "I am annoyed with my coach's bias against me" or "I am annoyed by my repetitive

189 injury," participants responded about the frequency of the event on a six-point Likert scale

190 ranging from 1 (never) to 6 (always). The Cronbach's $\alpha$ for these factors in the present study

191 ranged from 0.69 to 0.87 , indicating that the measure was reliable. Lu and colleagues (2012)

192 reported CSALSS with adequate concurrent and discriminant validity. Also, related research 
193 indicated that CSALSS correlated with global perceived stress (Chiu et al., 2016) which provides

194 evidence of predictive validity.

195 Negative Thoughts: A 30-item Automatic Thoughts Questionnaire (ATQ; Hollon \& Kendall,

196 1980) was applied to assess the frequency of occurrence for 30 types of thoughts in the previous

197 week. Participants rated each single negative self-statement on a 5-point Likert scale ranging

198 from 1 (not at all) to 5 (always). The ATQ was divided into four subscales: (a) Personal

199 Maladjustment and Desire for Change (PMADC) (e.g., I wish I were a better person); (b)

200 Negative Self-Concept (NSC) (e.g., I am a failure); (c) Giving Up/Helplessness (GU/H) (e.g., I

201 cannot finish anything); and (d) Negative Expectations (NE) (e.g., I cannot get things together).

202 All items represented experiences and thoughts of depression. Total scores for ATQ ranged from

20330 (no or a minor depression) to 150 (major depression). Hollon and Kendall (1980) reported

204 ATQ can differentiate depressed and non-depressed individuals. Also, ATQ has been validated

205 in Norwegian and found positively correlated with Beck Depression Inventory (Chioqueta \&

206 Stiles, 2004).

Burnout: An 11-item Athlete Burnout Questionnaire (ABQ; Lu et al., 2006) revised from 
208 Raedeke and Smith (2001) burnout scale was used to assess athletes' burnout. The ABQ was

209 divided into three subscales: emotional or physical exhaustion, sports devaluation, and a reduced

210 sense of accomplishment. Sample questions assessing emotional or physical exhaustion included

211 "I feel so tired from my training that I have trouble finding energy to do anything else." To assay

212 for a reduced sense of accomplishment, questions included "I am accomplishing many

213 worthwhile things in sports." Finally, to assess sports devaluation, questions included "The effort

214 I spend in sports would be better spent doing other things." Among all the items on the ABQ,

215 item 1 and 11 were reversed to score. To complete the ABQ, participants chose options on a

216 five-point Likert scale ranging from 1 (almost never) to 5 (always) to assess their experiences.

217 The higher the number participants identified, the higher the degree of burnout. The results were

218 internally consistent with scores of $0.85,0.86$, and 0.63 . In this research, we used a composite

219 score by adding the three subscales together. Lu and colleagues (2006) found ABQ positively

220 correlated with amotivation and negatively correlated with intrinsic motivation which provides

221 concurrent validity and discriminant validity. 


\section{Procedure}

223 After receiving approval from a local institutional review board (TSMH IRB No. 16-094-

224 B1), the research team contacted the coach or administrator of a target team and asked

225 permission to invite his/her team as participants. After accepting, we visited the target team one

226 hour before they finished their regular training. Before administering the questionnaire, we

227 explained the general purpose of the study, the methods to complete questionnaires, and the

228 rights associated with being a participant. To prevent social desirability effects, we informed

229 participants that this was a study to explore college students' life experiences, and there were no

230 right or wrong answers. Additionally, we asked participants to answer the questions as truthfully

231 as possible, and all responses would be confidential. After the briefing, participants who were

232 interested in participating in this study signed a consent form and completed the investigation

233 package. It took about 15 minutes to complete the questionnaires.

\section{Statistical Analyses}

\section{Preliminary Analyses}


236 First, a descriptive statistical analysis was applied to examine the properties of the collected

237 data, including skewness, kurtosis, means, standard deviations, outliers, and missing data for all

238 variables. The skewness $(0.10-1.38)$ and kurtosis $(-0.10-1.38)$ were found to be in an acceptable

239 range. Gender, age, and competition levels for all variables were examined using a $t$-test and

240 analysis of variance (ANOVA). The results indicated that there was a significant gender

241 difference in the subscales of "personal maladjustment and desire for change (PMADC)" and

242 "giving up/helplessness $(\mathrm{GU} / \mathrm{H})$ " for the ATQ. Therefore, in the subsequent hierarchical

243 regression analyses, gender was controlled for when examining the mediating effects of these

244 two factors.

245 Hypothesis Testing

246 A Pearson product-moment correlation analysis was used to examine the relationships of all

247 variables and provide information about whether the predictor variable (i.e., two types of life

248 stress), criterion variable (i.e., burnout), and mediating variables (i.e., four types of negative

249 thoughts) correlated. This analysis was conducted as a prerequisite analysis for testing mediator

250 effects (Baron \& Kenny, 1986, p. 1174). According to Baron and Kenny’s (1986) suggestions, 
251 we examined mediation by assuming that the following conditions were met: (a) two types of life

252 stress should predict four types of negative thoughts, (b) four types of negative thoughts should

253 predict burnout, and (c) two types of life stress should predict burnout. If all three conditions

254 were met, the subsequent mediating effects of four types of negative thoughts on the relationship

255 between two types of life stress and burnout were further analyzed.

256 A series of hierarchical regression analyses were applied to examine the main effects and

257 interaction effects. First, two types of life stress (general life stress and sport-specific stress) were

258 entered into the regression at the first step. Then, four types of negative thoughts were entered at

259 the second step. Two types of life stress and four types of negative thoughts were simultaneously

260 entered at the third step. The final test for mediation was to check whether two types of life stress

261 predicted burnout when four types of negative thoughts were controlled for. 
265 These results indicated that athlete burnout positively correlated with a total score for negative

266 thoughts, four types of negative thoughts, and two types of life stress. Among these correlations,

267 burnout correlated more significantly with two types of life stress than negative thoughts. Four

268 types of negative thoughts positively correlated with each other, as did two types of life stress.

(Insert Table 1 here)

of negative thoughts $(\beta=0.53 ; \beta=0.50 ; \beta=0.44 ; \beta=0.40, p<0.01)$ in Model 1. In Model 2, both sports-specific life stress $(\beta=0.54 ; \beta=0.54 ; \beta=0.54 ; \beta=0.54, p<0.01)$ and four types of

275 negative thoughts $(\beta=0.46 ; \beta=0.47 ; \beta=0.41 ; \beta=0.42, p<0.01)$ predicted burnout. Thus,

276 these results demonstrated that it these data can be further analyzed for mediating effects. Also,

277 as Table 3 illustrates, general life stress predicted four types of negative thoughts $(\beta=0.56 ; \beta=$

$2780.53 ; \beta=0.44 ; \beta=0.44, p<0.01)$ in Model 1. In Model 2 , both general life stress $(\beta=0.49 ; \beta=$ 
$0.22449 ; \beta=0.49 ; \beta=0.49, p<0.01)$ and four types of negative thoughts $(\beta=0.46 ; \beta=0.47 ; \beta$

$280=0.41 ; \beta=0.42, \mathrm{p}<0.01)$ predicted burnout. Thus, these results indicated that these data can be

281 further analyzed for mediating effects.

282

(Insert Tables $2 \& 3$ here)

283

Mediating effects of negative thoughts on the sports-specific life stress-burnout relationship

the sports-specific life stress-burnout relationship. The first block illustrates that personal

maladjustment and the desire for change (PMADC) mediated the sports-specific life stress-

burnout relationship ( $\beta=0.54$ changed to $\beta=0.41$ ). The second block demonstrates that negative

self-concept (NSC) mediated the sports-specific life stress-burnout relationship ( $\beta=0.54$

changed to $\beta=0.41)$. The third block demonstrates that giving up and helplessness $(\mathrm{GU} / \mathrm{H})$

291 fourth block shows that negative self-concept (NE) mediated the sports-specific life stress-

292 burnout relationship ( $\beta=0.54$ changed to $\beta=0.44)$. Sobel tests of PMADC, NSC, GU/H, and

293 NE were 6.86, 6.74, 5.63, and $5.81(\mathrm{z}>1.96$ and $p<0.05)$, respectively. 
294 Mediating effects of negative thoughts on the general life stress-burnout relationship

295 The lower part of Table 4 shows the mediating effects of four types of negative thoughts on

296 the general life stress-burnout relationship. The first block illustrates that personal maladjustment

297 and the desire for change (PMADC) mediated the general life stress-burnout relationship $(\beta=$

2980.49 changed to $\beta=0.34$ ). The second block demonstrates that negative self-concept (NSC)

299 mediated the sports-specific life stress-burnout relationship $(\beta=0.49$ changed to $\beta=0.34)$. The

300 third block shows that giving up and helplessness $(\mathrm{GU} / \mathrm{H})$ mediated the general life stress-

301 burnout relationship ( $\beta=0.49$ changed to $\beta=0.39$ ). The fourth block shows that negative self-

302 concept (NE) mediated the general life stress-burnout relationship $(\beta=0.49$ changed to $\beta=0.38)$.

303 Sobel tests for PMADC, NSC, GU/H, and NE $\mathrm{p}$ were 7.03, 7.02, 5.68, and $5.87(\mathrm{z}>1.96$ and $p<$

304 0.05), respectively.

305

(Insert Table 4 here)

306

Discussion

307

To determine whether negative thoughts might influence the stress-burnout relationship

308 for athletes, this study examined the relationships among athletes' life stress, negative thoughts, 
309 and burnout; and the potential mediating effects of negative thoughts on the life stress-burnout

310 relationship. Results indicated that two types of athletes' life stress, four types of negative

311 thoughts, and burnout were all positively correlated. Also, four types of negative thoughts

312 mediated a general life- and sports-specific stress-burnout relationship.

313 Our results provide several theoretical implications for researchers. By incorporating

314 negative thoughts into Smith's (1986) cognitive-affective model of athletic burnout, our study

315 advances our knowledge of how negative thoughts underlie the life stress-burnout relationship

316 for athletes. We found that the association between athletes' life stress and burnout was partially

317 explained by negative thoughts. Past research examining the mechanisms underlying the stress-

318 burnout relationship generally found that positive variables, such as self-efficacy (e.g., Schwazer

319 \& Hallum, 2008), optimism (Gustafsson, \& Skoog, 2012), teaching strategy (e.g., Ben-Ari et al.,

320 2003), hardiness (e.g., Chan, 2003), coping and social support (e.g., Koeske \& Koeske, 1989;

321 Raedeke \& Smith, 2004), or perceived accomplishments (e.g., Koeske \& Koeske, 1989), mediate

322 or moderate the stress-burnout relationship. Our study provides new evidence that negative 
323 thoughts can change the stress-burnout relationship. These results should prompt future research

324 to examine how positive and negative variables influence the stress-burnout relationship.

325 The mediating effect of negative self-concept (NSC) on the two types of life stress-

326 burnout relationships suggests that there is a role for the self-concept in predicting athletes'

327 motivation, affect, and behavior (Gallia, \& Gonzalez, 2014). Past research found that when

328 athletes had low self-esteem, they either adopted self-handicapping strategies in sports (Finez, \&

329 Sherman, 2012) or were susceptible to developing eating disorders (McLester, Hardin, \& Hoppe,

330 2014). Thus, it is important to monitor athletes' self-related thought patterns. Additionally,

331 coaches, parents, and sports professionals need to build a positive motivational culture in which

332 athletes' self-concept will not be threatened in losing situations (Smith, Smoll, \& Cumming,

333 2007).

334 The effect of giving up and helplessness $(\mathrm{GU} / \mathrm{H})$ on the relationship between two types of

335 life stress and burnout prompted us to examine athletes' causal explanation about their failure in

336 sports. Why one gives up and displays helplessness derives from attributing failure to internal

337 and stable reasons (Weiner, 1972). As previously stated, female athletes tend to attribute success 
338 to external, unstable, and uncontrollable causes, while attributing failure to internal, stable, and

339 controllable reasons (Green \& Holeman, 2004). Therefore, how to change athletes' thought

340 patterns and attribution retraining for those with self-defeating attribution is key for coaches and

341 sport psychology consultants.

343 life stress and burnout imply that when athletes tend to be negative about their future, they will

344 increase the life stress-burnout relationship. Although no specific investigation focused on the

345 sources of negative expectations in sports, it has been shown that social agents, such as coaches,

346 parents, peers, and teachers, play a significant role in formulating athletes' expectations

347 (Weinberg \& Gould, 2015). Also, Bandura's (1997) self-efficacy theory indicated that personal

348 accomplishments, vicarious experiences, verbal persuasion, and physiological responses are the

349 sources of efficacy expectations. Thus, developing strategies to facilitate athletes' positive

350 expectations and reduce negative expectations will be effective for reducing athlete burnout.

351 The mediating effect of personal maladjustment and the desire for change (PMADC) on

352 the life stress-burnout relationship suggests that such depressogenic thoughts may increase 
353 athletes' burnout. As previously stated, athletes encounter many stressors from either life or

354 sports. Literature has suggested that chronic stress leads to depression (Risch et al., 2009),

355 increasing hopelessness and suicidal ideation (Glick et al., 2012). Furthermore, it has been found

356 that depressed adolescent athletes are susceptible to doping (Blank et al., 2016). Therefore,

357 future studies should examine how athletes' personal maladjustment and desire for change

358 influence unhealthy behaviors, motivations, and overall well-being.

359 In addition, the sources of athlete burnout are worthy of discussion. The extant sport

360 burnout literature suggests that environmental factors (e.g., excessive training, stressful social

361 relationship, negative performance demands, low social support), personal factors (e.g.,

362 perfectionism, trait anxiety, low autonomy), and motivational factors (e.g., ego orientation,

363 performance motivational climate) are causes of athletic burnout (Gould \& Whitley, 2009;

364 Gustafsson, Kentta, \& Hasmen, 2011). Thus, our results add to this body of knowledge by

365 showing that athletes' thought patterns can be associated with burnout. Specifically, Smith's

366 (1986) cognitive-affective model of athletic burnout emphasizes the role of the cognitive process

367 in the stress-burnout relationship. Therefore, how another type of thought, such as positive 
368 thoughts (Bryant \& Baxter, 1997), influences an athlete's life stress-burnout relationship is

369 worthy of investigation in the future. The effects of positive thoughts (e.g., positive daily

370 functioning, positive self-evaluation, positive evaluation of self, and positive future expectation)

371 can be new directions for athlete burnout research.

378 indicated that there are many types of life stressors, such as work and family (Bacharach,

379 Bamberger, \& Conley, 1991), organizational stress (Arnold, Fletcher, \& Daniels, 2013), and both

380 general and sports-specific life stressors (Lu et al., 2012). Hence, how different types of life

381 stressors interact with negative thoughts to influence an individual's health and well-being is

382 another direction for future research. 
383 Limitations and Future Suggestions

384 There are several limitations that should be addressed here. First, our study is cross-

385 sectional in nature. Therefore, the causes and effects of the stress-burnout relationship and the

386 mediating effect of negative thoughts on the stress-burnout relationship are not warranted. We

387 suggest that future studies adopt a longitudinal approach to observe how life stress associated

388 with negative thoughts subsequently predicts burnout (Eklund \& DeFreese, 2015). Second, these

389 data were collected from Taiwanese student-athletes. Thus, our results may not be generalizable

390 to different cultures. We recommend that researchers adopt a similar approach for examining the

391 relationships among life stressors, negative thoughts, and burnout in a different culture.

392 Furthermore, our sample consisted of Division I college athletes. Whether our results could be

393 generalized to other athletes, such as professional athletes or junior athletes, needs to be

394 examined. Moreover, since we adopted Smith's (1986) cognitive-affective model of athletic

395 burnout as a guiding framework for our study, it is possible that the three sets of variables (life

396 stress, negative thoughts, and burnout) could be causally related in several ways. For example,

397 life stress could precipitate burnout, which could lead to negative thoughts; and burnout plays a 
398 mediating role in the model. We suggest that future studies examine these potential models by

399 using related theoretical frameworks. Last, we used hierarchical regression to analyze the

400 mediating effects of negative thoughts on life stress-burnout relationship, it is suggested that

401 other statistical techniques, such as structural equation modeling (SEM), path analysis and factor

402 analysis, can be applied to examine the causal effects of the model depends on the theories and

403 aims of the study (Musil, Jones, \& Warner, 1998).

\section{Conclusions}

405 Burnout is a significant issue for athletes, administrators, parents, coaches, and sport

406 psychology consultants because it can be detrimental to a youth's psychosocial development and

407 well-being. Athletes may have different sources of burnout. Our study found that both life stress

408 and negative thoughts interact to influence athletes' burnout. We consider our study to be a

409 starting point for researchers to examine the role of negative thoughts in athlete burnout. We

410 suggest sports professionals should be aware of athletes' burnout and build a healthy competitive

411 environment for youth athletes. 


\section{References}

413 Allen, M.S. (2012). A systematic review of content themes in sport attribution research: 19522011. International Journal of Sport and Exercise Psychology, 10(1), 1-8.

415 Arpin-Cribbie, C.A., \& Cribbie, R. A. (2007). Psychological correlates of fatigue: Examining

416 depression, perfectionism, and automatic negative thoughts, Personality and Individual

417 Differences 43, 1310-1320.

418 Arvinen-Barrow, M., Clement, D., Hamson-Utley, J.J., Kamphoff, C., Zakrajsek, R., Lee, S.,

419 Hemmings, B., Lintunen, T., \& Martin, S.B. (2016). Athletes’ expectations about sport-

420 injury rehabilitation: A cross-cultural study.

421 Bacharach, S. B., Bamberger, P., \& Conley, S. (1991). Work-home conflict among nurses and

422 engineers: mediating the impact of role stress on burnout and satisfaction at work. Journal of Organizational Behavior, 12, 39-53.

424 Bandura, A. ( 1997 ) Self-efficacy: the exercise of control. New York: W. H. Freeman. 
425 Barron, R. M. \& Kenny, D. A. (1986). The moderator-mediator variable distinction in social Universities Press. Journal of Stress Management, 10, (2), 173-195. constructs as predictors of doping susceptibility in adolescent athletes. Asian Journal of Sports Medicine, 7(4), 1-9. doi: 10.5812/asjsm.35024. 
438 Bryant, F.B., \& Baxter, W. J. (1997). The structure of positive and negative automatic cognition.

$439 \quad$ Journal of Cognition and Emotions, 3, 225-258.

$440 \quad$ http://dx.doi.org/10.1080/026999397379908

441 Chan, D. W. (2003). Hardiness and its role in the stress-burnout relationship

442 among prospective Chinese teachers in Hong Kong, Teaching and Teacher Education 19,

$443 \quad 381-395$.

444 Chang, Y. K., Ho, L. A., Lu, F. J. H., Ou, C. C., Song, T. F., \& Gill, D. (2014). Self-talk and

445 softball performances: The role of self-talk nature, motor task characteristics, and self-

446 efficacy in novice softball players. Psychology of Sport and Exercise,15,135-149.

$447 \quad$ http://dx.doi.org/10.1016/j.psychsport.2013.10.004.

448 Chioqueta, A. P., \& Stiles, T.C. (2004). Norwegian Version of the Automatic Thoughts

449 Questionnaire: A Reliability and Validity Study. Cognitive Behavioural Therapy, 33(2), 79-

82. DOI 10.1080/16506070310016031. 
451 Chiu, Y.S, Lu, F.J.H., Lin, J.H., Nien, C.L., Hsu, Y.W., \& Liu, H.Y. (2016). $\quad$ Psychometric

452

453

454 Clark, D., \& Goosen, T. (2009). The mediating effects of coping strategies in the relationship

Dimmock, J.A., Grove, J.R., \& Eklund, R.C. (2005). Reconceptualising team identification: New and attributions. Journal of Applied Sports Psychology, 18, 219-239.

Cresswell, S., \& Eklund, R.C., (2006). The nature of player burnout in rugby: key characteristics Dimmock, J.A., Grove, J.R., \& Eklund, R.C. (2005). Reconceptualising team identification: New dimensions and their relationship to intergroup bias. Group Dynamics: Theory, Research,

Eklund, R., \& DeFreese, J.D. (2015). Athlete Burnout: What we know, what we could know, and and Practice, 9, 7586.

\section{4}

Finez, L., \& Sherman, D.K. (2012). Train in Vain: The Role of the Self in Claimed Self- 
466 Franciscoa, C. Arceb, C., Vílcheza, M., \& Valesc, A. ( 2016).Antecedents and consequences of burnout in athletes: Perceived stress and depression. International Journal of Clinical and Health Psychology, 16, 239-246.

469 Gernigon, C., \& Delloye, J.B. (2003). Self-efficacy, causal attribution, and track athletic performance following unexpected success or failure among elite sprinters. The Sport Psychologist, 17, $55-76$.

Gil, D.M., Williams, D.A., Keefe, F.J., \& Beckham, J.C. (1990). The relationship of negative thoughts to pain and psychological distress. Behavior Therapy, 21(3), 349-362.

474 Glick, I.D., Stillman, M.A., Reardon, C.L., \& Ritvo, E.C. (2012). Managing psychiatric issues in elite athletes, The Journal of Clinical Psychology, 73(5):640-644. DOI: $\underline{10.4088 / J C P .11 r 07381}$

477 Gould, D., Tuffey, S., Udry, E., \& Loehr, J. (1997). Burnout in competitive junior tennis players: III. Individual differences in the burnout experience. The Sport Psychologist, 11, 257-276.

Gould, D., \& Whitley, M.A. (2009). Sources and consequences of athletic burnout among college athletes. Journal of Intercollegiate Sports, 2, 16-30. 
481 Green, T.D., \& Holeman, S. (2004). Athletes' attributions for team performance: A theoretical

482 test across sports and genders. Social Behavior and Personality: an International Journal,

484 Gustafsson, H., Hassmen, P., Kenta, G., \& Johansson, M. (2008). A qualitative analysis of

487 Gustafsson, H., Kentta, G., Hassmen, P., \& Lundqvist, C. (2007). Prevalence of burnout in

488 adolescent competitive athletes. The Sport Psychologist, 20, $21-37$.

489 Gustafsson, H. \& Skoog, T. (2012). The mediational role of perceived stress in the relation

490 between optimism and burnout in competitive athletes. Anxiety, Stress, and Coping, 25(2),

$491 \quad 183-199$.

492 Gustafsson, H., Skoog, T., Podlog, Lundquist, Wagnsson, S. (2013). Hope and athlete burnout:

493 Stress and affect as mediators. Psychology of Sport and Exercise, 14, 640-649. 
494 Hatzigeorgiadis, A., Zourbanos, N., Galanis, E., \& Theodorakis, Y. (2011). Self-talk and sports performance: A meta-analysis. Perspectives on Psychological Science, 6, 348-356. http://dx.doi.org/10.1177/1745691611413136.

497 Hollon, S. D. \& Kendall, P. C. (1980). Cognitive self-statements in depression: Development of and Automatic Thoughts Questionnaire. Cognitive Therapy and Research. 4. 383-395.

Koeske, G. F., \& Koeske, R.D. (1989). Work load and burnout: Can social support and perceived accomplishment help? Social Work, 243-248. Therapy Research, 37, 160-172. Education Journal, 39 (3), 83 94.[in Chinese]. in Physical Education and Exercise Science, 16(4), 254-267. 
509 Lu, F.J.H., Lee, W.P., Chang, Y.K., Chou, C.C., Hsu, Y.W., Lin, J.H., \& Gill, D.L. (2016).

510 Interaction of athletes' resilience and coaches' social support on the stress-burnout

511 relationship: A conjunctive moderation perspective, Psychology of Sport and Exercise 22,

$512 \quad 202-209$.

513 Martens, R., Burton, D., Vealey, R.S., Bump, L.A., \& Smith, D.E. (1990). Development and

514 validation of the Competitive State Anxiety Inventory-2. In R. Martens, R.S. Vealey, \& D.

515 Burton, Competitive anxiety in sport (pp. 117-190). Champaign, IL: Human Kinetics

516 McLester, C.N., Hardin, R., \& Hoppe, S. (2014). Susceptibility to eating disorders among

517 collegiate female student-athletes. Journal of Athletic Training, 49(3):406-410 doi:

$518 \quad 10.4085 / 1062-6050-49.2 .16$

519 Musil, C. M., Jones, S. L., \& Warner, C.D. (1998). Structural equation modeling and its

520 relationship to multiple regression and factor analysis. Research in Nursing and Health, 21,

$521 \quad 271-281$.

522 Raedeke, T. D., \& Smith, A. L. (2001). Development and preliminary validation of a measure of

523 athlete burnout measure. Journal of Sport and Exercise Psychology, 23, 281-306. 
524 Raedeke, T. D., \& Smith, A. L. (2004). Coping resources and athlete burnout: An examination of stress-mediated and moderation hypothesis. Journal of Sport and Exercise Psychology, 26, 525-541.

531 Schwarzer, R. \& Hallum, S. (2008). Perceived Teacher Self-Efficacy as a Predictor of Psychology, 8, 36-50. 
539 Stiles, T. C., \& Gotestam, K. G. (1989). The role of automatic negative thoughts I the

540 development of dysphoric mood: An analog experiment. Cognitive Therapy and Research,

$541 \quad 13(2), 161-170$.

542 Suhr, J.A., \& Gunstad, A. (2004). Diagnosis threat": The effect of negative expectations on

543 cognitive performance in head injury. Journal of Clinical and Experimental

$544 \quad$ Neuropsychology, 24(4), $448 \pm 457$.

545 Weiner, B. (1972). Theories of motivation: From mechanism to cognition. Chicago: Markham.

546 Weinberg, R., \& Gould, D. (2015). Foundations of sport and exercise psychology (6 $\left.{ }^{\text {th }}\right)$ Human

$547 \quad$ Kinetics.

548 Zourbanos, N., Hatzigeorgiadas, N., Tsiakaras, N., Chroni, S., \& Theodorakis, Y. (2010). A

549 multimethod examination of the relationship between coaching behavior and athletes'

$550 \quad$ inherent self-talk. Journal of Sport \& Exercise Psychology, 32, 764-785. 


\section{Table 1 (on next page)}

Correlation matrix and descriptive statistics for study variables 
1 Table 1. Correlation matrix and descriptive statistics for study variables.

\begin{tabular}{|c|c|c|c|c|c|c|c|c|}
\hline & ABQ & ATQ & PMADC & NSC & $\mathrm{GU} / \mathrm{H}$ & $\mathrm{NE}$ & $\begin{array}{l}\text { Sport } \\
\text { Stress }\end{array}$ & $\begin{array}{l}\text { General } \\
\text { Stress }\end{array}$ \\
\hline 1. $\mathrm{ABQ}$ & 1.00 & $.47^{* *}$ & $.46^{* *}$ & $.47^{* *}$ & $.41^{* *}$ & $.42^{* *}$ & $.54^{* *}$ & $.49^{* *}$ \\
\hline 2. ATQ & & 1.00 & $.86^{* *}$ & $.88^{* *}$ & $.96^{* *}$ & $.95^{* *}$ & $.49^{* *}$ & $.52^{* *}$ \\
\hline 3. PMADC & & & 1.00 & $.79^{* *}$ & $.74^{* *}$ & $.69^{* *}$ & $.53^{* *}$ & $.56^{* *}$ \\
\hline 4. NSC & & & & 1.00 & $.79^{* *}$ & $.80^{* *}$ & $.50^{* *}$ & $.53^{* *}$ \\
\hline 5. GU/H & & & & & 1.00 & $.92^{* *}$ & $.44^{* *}$ & $.44^{* *}$ \\
\hline 6. NE & & & & & & 1.00 & $.40^{* *}$ & $.44^{* *}$ \\
\hline 7. Sport Stress & & & & & & & 1.00 & $.68^{* *}$ \\
\hline 8. Gen. Stress & & & & & & & & 1.00 \\
\hline Mean & 2.48 & 1.86 & 2.36 & 1.92 & 1.76 & 1.62 & 2.61 & 2.43 \\
\hline$S D$ & 0.76 & 0.65 & 0.77 & 0.73 & 0.70 & 0.67 & 0.85 & 0.96 \\
\hline$\alpha$ & .89 & .96 & .84 & .76 & .91 & .94 & .89 & .90 \\
\hline
\end{tabular}

2 Note. ${ }^{\mathrm{a}} \mathrm{ABQ}=$ athlete burnout; $\mathrm{ATQ}=$ total score of automatic negative thoughts; $\mathrm{PMADC}=$

3 personal maladjustment and desire for change, $\mathrm{NSC}=$ negative self-concept, $\mathrm{GU} / \mathrm{H}=$ giving

4 up/helplessness, NE=negative expectations; Sport Stress=sport-specific life stress; Gen. Stress=

5 general-life stress; ${ }^{*} p<.05,{ }^{* *} p<.01$.

6

7

8

9

10

11

12

13

14

15

16

17

18

19

20

21

22 
Table 2 (on next page)

Simple regression of sport- life stress and negative thoughts on burnout 
1 Table 2. Simple regression of sport- life stress and negative thoughts on burnout.

3

4 Variables

5

7 Regression 1

8

9

10 Regression 2

11

12

13 Negative Thoughts. $46^{* *} \quad .21^{* *} \quad .47^{* *} \quad .22^{* *} \quad .41^{* *} \quad .16^{* *} \quad .42^{* *} \quad .18^{* *}$

14 Note: aThe dependent variables of regression 1 was negative thoughts, and regression 2 was

15 burnout. All abbreviations were as indicated in Table 1. ${ }^{\text {b** }}, p<.01$. 


\section{Table 3 (on next page)}

Simple regression of general-life stress and negative thoughts on burnout. 
1 Table 3. Simple regression of general-life stress and negative thoughts on burnout.

4

5

6

7 Regression 1

8

9

10 Regression 2

11 Gen-life stress $.49 * * \quad .24 * * \quad .49 * * \quad .24 * * \quad .49 * * \quad .24 * * \quad .49 * * \quad .24 * *$

12

13 Negative Thoughts.46** $.21 * * \quad .47^{* *} \quad .22^{* *} \quad .41^{* *} \quad .16^{* *} \quad .42^{* *} \quad .18^{* *}$

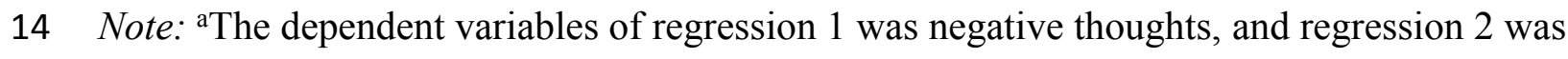

15 burnout. ${ }^{\mathrm{b}}$ All abbreviations were as indicated in Table $1 .{ }^{* *}, p<.01$. 16

17 


\section{Table 4(on next page)}

Mediating models of negative thoughts on life stress-burnout relationships. 
Table 4. Mediating models of negative thoughts on life stress-burnout relationships.

\begin{tabular}{|c|c|c|c|c|c|c|c|c|c|c|c|c|}
\hline \multirow{5}{*}{$\begin{array}{l}\text { Variables } \\
\text { Model } 2 \\
\beta \quad \Delta R^{2}\end{array}$} & \multirow[b]{5}{*}{$\Delta R^{2}$} & \multicolumn{11}{|c|}{ Mediating Model } \\
\hline & & \multicolumn{3}{|c|}{ PMADC } & \multicolumn{4}{|c|}{$\mathrm{NSC}$} & \multicolumn{2}{|c|}{$\mathrm{GU} / \mathrm{H}$} & \multicolumn{2}{|r|}{$\mathrm{NE}$} \\
\hline & & \multicolumn{2}{|r|}{ Model 1} & Model 2 & \multicolumn{2}{|c|}{ Model 1} & \multicolumn{2}{|c|}{ Model 2} & \multirow[t]{3}{*}{ Model 1} & \multirow[t]{3}{*}{ Model 2} & \multirow{3}{*}{\multicolumn{2}{|c|}{ Model 1}} \\
\hline & & & $\beta \quad \Delta R^{2}$ & $\beta \quad \Delta R^{2}$ & $\beta$ & $\Delta R^{2}$ & $\beta$ & $\Delta R^{2}$ & & & & \\
\hline & & $\beta$ & $\Delta R^{2} \quad \beta$ & $\Delta R^{2}$ & & & & & & & & \\
\hline \multirow{2}{*}{\multicolumn{2}{|c|}{$\begin{array}{l}\text { Step } 1 \\
\quad \text { Sport stress }\end{array}$}} & \multicolumn{3}{|c|}{$.29 * *$} & \multicolumn{3}{|c|}{$.29 * *$} & & \multicolumn{2}{|c|}{$.29 * *$} & \multicolumn{2}{|c|}{$.29 * *$} \\
\hline & & & $.54 * *$ & $.41 * *$ & $.54 * *$ & & $.41 * *$ & & $.54 * *$ & $.45 * *$ & $.54 * *$ & $.44 * *$ \\
\hline \multirow{2}{*}{\multicolumn{13}{|c|}{$\begin{array}{l}\text { Step } 2 \\
34^{* *}\end{array}$}} \\
\hline & & & & & & & & & & & & \\
\hline$P M A D C$ & & & & $.24 * *$ & & & & & & & & \\
\hline$N S C$ & & & & & & & $.26 * *$ & & & & & \\
\hline$G U / H$ & & & & & & & & & & $.21 * *$ & & \\
\hline$N E$ & & & & & & & & & & & & $.25 * *$ \\
\hline \multirow{2}{*}{\multicolumn{2}{|c|}{$\begin{array}{l}\text { Step } 1 \\
\quad \text { General stress }\end{array}$}} & \multicolumn{3}{|c|}{$.24^{* *}$} & \multicolumn{3}{|c|}{$.24 * *$} & \multicolumn{3}{|c|}{$.24 * *$} & \multicolumn{2}{|c|}{$.24 * *$} \\
\hline & & & $.49 * *$ & $.34 * *$ & $.49 * *$ & & $.34 * *$ & & $.49 * *$ & $.39 * *$ & $.49 * *$ & $.38 * *$ \\
\hline Step 2 & & & & $.28 * *$ & & & & $.30 * *$ & & $.28 * *$ & & . \\
\hline \multicolumn{13}{|l|}{$29 * *$} \\
\hline PMADC & & & & $.27 * *$ & & & & & & & & \\
\hline$N S C$ & & & & & & & $.29 * *$ & & & & & \\
\hline$G U / H$ & & & & & & & & & & $.24 * *$ & & \\
\hline$N E$ & & & & & & & & & & & & $.26 * *$ \\
\hline
\end{tabular}

2 Note: ${ }^{a}$ The upper part was the mediation analysis of negative thoughts on sport-specific life stress-burnout relationship; bSobel 33 tests of PMADC, NSC, GU/H and NE were 6.86, 6.74, 5.63 and $5.81(\mathrm{z}>1.96$ and $\mathrm{p}<.05)$, respectively. ${ }^{\mathrm{c}}$ The lower part was 


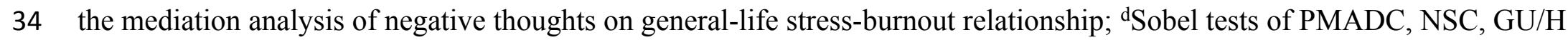
35 and NE $\mathrm{p}$ were 7.03, 7.02, 5.68 and $5.87(\mathrm{z}>1.96$ and $\mathrm{p}<.05)$, respectively. ${ }^{\mathrm{e}} \mathrm{All}$ abbreviations were as indicated in Table 1. $36 \mathrm{f} * *, p<.01$. 